\title{
A New Achievable Rate Region for the Discrete Memoryless Multiple-Access Channel with Feedback
}

\author{
Ramji Venkataramanan \\ Dept. of Electrical Engineering \\ Stanford University, USA \\ Email:vramji@stanford.edu
}

\author{
S. Sandeep Pradhan \\ Dept. of Electrical Engineering \& Computer Science \\ University of Michigan, Ann Arbor, USA \\ Email: pradhanv@eecs.umich.edu
}

\begin{abstract}
A single-letter achievable rate region for the twouser discrete memoryless multiple-access channel is proposed. The rate region includes the Cover-Leung region [1], and it is shown that the inclusion is strict. The proof uses a block-Markov superposition strategy based on the observation that the messages of the two users are correlated given the feedback. The rates of transmission are too high for each encoder to decode the other's message directly using the feedback, so they transmit correlated information in the next block in order to learn the the message of each other. They then cooperate in the following block to resolve the residual uncertainty of the decoder. Our scheme may be viewed as a natural generalization of the Cover-Leung scheme with a delay of one extra block and a pair of additional auxiliary random variables. The scheme can also be extended to obtain larger rate-regions with more auxiliary random variables.
\end{abstract}

\section{INTRODUCTION}

The two-user discrete memoryless multiple-access channel (MAC) is shown in Figure 1 The channel has inputs $X, Y$, output $Z$ and is characterized by the conditional law $P_{Z \mid X Y}$. The capacity region for this channel without feedback $\left(S_{1}\right.$ and $S_{2}$ open in Figure (1) was determined by Ahlswede [2] and Liao [3]. Gaarder and Wolf [4] demonstrated that feedback can enlarge the capacity region of this channel using the example of a binary erasure MAC. Cover and Leung [1] then established a single-letter achievable rate region for a discrete memoryless MAC with feedback. The Cover-Leung (C-L) region was shown to be the feedback capacity region for a class of discrete memoryless MACs [5]. However the $\mathrm{C}-\mathrm{L}$ region is smaller than the feedback capacity in general, the white Gaussian MAC being a notable example [6]. The capacity region of the MAC with feedback was characterized by Kramer [7], [8] in terms of directed information. This is a 'multi-letter' characterization and the existence of a singleletter capacity characterization for the discrete memoryless MAC with feedback remains an open question. Recently, a single-letter achievable region that includes the $\mathrm{C}$-L region was reported in [9].

The basic idea behind the Gaarder-Wolf and the C-L schemes is the following. Each message pair is conveyed to the decoder over two successive blocks of transmission. In

\footnotetext{
This work was supported by NSF Grant (CAREER) CCF-0448115.
}

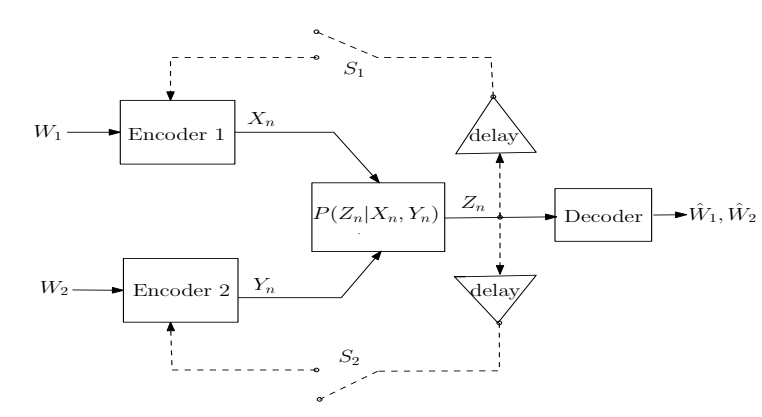

Fig. 1. The multiple-access channel. When $S_{1}, S_{2}$ are closed there is feedback to both encoders.

the first block, the two encoders transmit messages at rates outside the no-feedback capacity region. At the end of this block, the decoder cannot decode the message pair; however, each encoder can decode the message of the other using the feedback (the rates must be low enough to ensure this). The two encoders can now cooperate and send a common message to resolve the decoder's uncertainty in the next block. This procedure is repeated over several blocks with fresh information superimposed over resolution information in every block. This block-Markov superposition scheme yields rates outside the no-feedback capacity region. It was shown in [5] that this coding strategy is optimal when the channel is such that $X=g(Y, Z)$ and $Y=h(X, Z)$ for some functions $g, h$.

In the C-L strategy described above, suppose that the rates are too high for the encoders to decode the message of one another using the feedback. In this case, encoder 1 has its own message plus a list of possible messages for encoder 2 . This list, created from all the $Y$ sequences that are jointly typical with the known $(X, Z)$ sequence pair, is clearly smaller than the original message set of encoder 2 . Similarly, encoder 2 has a list of all possible messages for encoder 1 . Thus at the end of the first block, the encoders have correlated information they wish to transmit over the next block. Note that this is more general than the C-L scheme which imposes perfect correlation between the encoders' messages given a block of channel output. Transmission of correlated sources and correlated message graphs over the MAC was studied in 
[10] and [11], respectively. We use these ideas to develop a new block-Markov superposition coding scheme. This scheme yields a single-letter rate region that includes the $\mathrm{C}$ - $\mathrm{L}$ region. We show that the inclusion is strict using the example of the AWGN MAC.

\section{Preliminaries and Main Result}

We use uppercase letters to denote random variables, lowercase for their realizations and bold-face notation for random vectors. Unless otherwise stated, all vectors have length $N$. Thus $\mathbf{A} \triangleq A^{N} \triangleq\left(A_{1}, \ldots, A_{N}\right)$. For any $\alpha$ such that $0<$ $\alpha<1, \bar{\alpha} \triangleq 1-\alpha$. The typical set of a random variable with pmf $P$ is denoted $T(P)$. Logarithms are with base 2, and entropy and mutual information are measured in bits.

Consider a two-user discrete memoryless multiple-access channel with input alphabets $\mathcal{X}$ and $\mathcal{Y}$, output alphabet $\mathcal{Z}$ defined by the probability mass functions $P_{Z \mid X Y}(. \mid x, y)$ for all $x \in \mathcal{X}, y \in \mathcal{Y}$. The channel is memoryless, i.e., it satisfies

$$
P\left(Z_{n} \mid X^{n}, Y^{n}, Z^{n-1}\right)=P\left(Z_{n} \mid X_{n}, Y_{n}\right), n=1,2, \ldots
$$

There is feedback to both encoders $\left(S_{1}\right.$ and $S_{2}$ are both closed in Figure 1].

Definition 1: An $\left(N, 2^{N R_{1}}, 2^{N R_{2}}\right)$ code with block length $N$ and rates $\left(R_{1}, R_{2}\right)$ for a MAC with feedback consists of

1) A sequence of mappings for each encoder:

$$
\begin{aligned}
& e_{1 n}:\left\{1, \ldots, 2^{N R_{1}}\right\} \times \mathcal{Z}^{n-1} \rightarrow \mathcal{X}, \quad n=1, \ldots, N \\
& e_{2 n}:\left\{1, \ldots, 2^{N R_{2}}\right\} \times \mathcal{Z}^{n-1} \rightarrow \mathcal{Y}, \quad n=1, \ldots, N
\end{aligned}
$$

2) A decoder mapping given by

$$
g: \mathcal{Z}^{N} \rightarrow\left\{1, \ldots, 2^{N R_{1}}\right\} \times\left\{1, \ldots, 2^{N R_{2}}\right\}
$$

Assuming the messages $\left(W_{1}, W_{2}\right)$ are drawn uniformly from the set $\left\{1, \ldots 2^{N R_{1}}\right\} \times\left\{1, \ldots 2^{N R_{2}}\right\}$, the error probability is

$P_{e, N}=\frac{\sum_{m_{1}=1, m_{2}=1}^{2^{N R_{1}}, 2^{N R_{2}}} \operatorname{Pr}\left(g(\mathbf{Z}) \neq\left(m_{1}, m_{2}\right) \mid W_{1}, W_{2}=m_{1}, m_{2}\right)}{2^{N R_{1}} 2^{N R_{2}}}$

A rate pair $\left(R_{1}, R_{2}\right)$ is achievable for the discrete memoryless MAC with feedback if there exists a sequence of $\left(N, 2^{N R_{1}}, 2^{N R_{2}}\right)$ codes such that $P_{e, N} \rightarrow 0$ as $N \rightarrow \infty$. The closure of all achievable rate pairs is the capacity region with feedback.

Cover and Leung established the following theorem characterizing an achievable rate region for the MAC with feedback. Cover-Leung Region: Consider a joint distribution $P$ of the form $P_{U X Y Z}=P_{U} P_{X \mid U} P_{Y \mid U} P_{Z \mid X Y}$, where $P_{Z \mid X Y}$ is fixed by the channel and $U$ is a discrete random variable with cardinality $\min \{|\mathcal{X}| \cdot|\mathcal{Y}|+1,|\mathcal{Z}|+2\}$. For any such $P$, the following rate pairs $\left(R_{1}, R_{2}\right)$ are achievable.

$$
\begin{gathered}
R_{1}<I(X ; Z \mid Y U), \quad R_{2}<I(Y ; Z \mid X U), \\
R_{1}+R_{2}<I(X Y ; Z)
\end{gathered}
$$

The following theorem is the main result of this paper.

Theorem 1: For the MAC described above consider any joint distribution of the form

$$
P_{U W A B X Y Z}=P_{U} P_{W A B} P_{X \mid U W A} P_{Y \mid U W B} P_{Z \mid X Y}^{c h}
$$

with $U, W, A, B$ are discrete random variables such that:

1) There exists a function $f: \mathcal{Z} \times \mathcal{A} \times \mathcal{B} \times \mathcal{U} \times \mathcal{W} \rightarrow \mathcal{W}$ such that

$$
P_{W}(k)=\sum_{\substack{(z, a, b, u, w): \\ f(z, a, b, u, w)=k}} P_{Z A B U W}(z, a, b, u, w)
$$

where $P_{Z A B U W}$ is the marginal derived from (2).

2) There exist conditional probability distributions $Q_{\bar{A} \mid Z X A B U W}$ (denoted $Q^{1}$ ) and $Q_{\bar{B} \mid Z Y A B U W}$ (denoted $Q^{2}$ ) such that for all $(\bar{a}, \bar{b}) \in \mathcal{A} \times \mathcal{B}$ and all $(z, a, b, u, w) \in \mathcal{Z} \times \mathcal{A} \times \mathcal{B} \times \mathcal{U} \times \mathcal{W}$

$$
\begin{gathered}
\sum_{x, y} Q^{1}(\bar{a} \mid z, x, a, b, u, w) Q^{2}(\bar{b} \mid z, y, a, b, u, w) \\
\cdot P_{X Y \mid Z A B U W}(x, y \mid z, a, b, u, w) \\
=P_{A B \mid W}(\bar{a}, \bar{b} \mid f(w, u, a, b, z)),
\end{gathered}
$$

where $P_{X Y \mid Z A B W}$ and $P_{A B \mid W}$ are marginals derived from (2).

For such a joint distribution, the following rate-region is achievable.

$$
\begin{aligned}
R_{1}<\min \{I(X ; Z \mid Y B W U), \\
I(X ; Z \mid Y A B W U)+I(A ; Z \mid B W U)+I(U ; Z)\} \\
R_{2}<\min \{I(Y ; Z \mid X A W U), \\
\quad I(Y ; Z \mid X A B W U)+I(B ; Z \mid A W U)+I(U ; Z)\} \\
R_{1}+R_{2}<I(X Y ; Z \mid U W)+I(U ; Z)
\end{aligned}
$$

\section{A. Overview of Coding Scheme}

Consider $B$ blocks of transmission, with a fresh pair of messages in each block. Let $\left(m_{1 b}, m_{2 b}\right)$ denote the messages, and $\mathbf{X}_{b}, \mathbf{Y}_{b}$ the input sequences for block $b$. The input $\mathbf{X}_{b}$ has three components- $\mathbf{U}_{b}, \mathbf{A}_{b}$ and a fresh message from user 1. $\mathbf{Y}_{b}$ comprises $\mathbf{U}_{b}, \mathbf{B}_{b}$ and a fresh message from user 2.

We note that the messages transmitted by the two users in any block are independent; however, conditioned on the output of that block, the messages are correlated. Consider the block $(b-1)$, at the end of which the decoder as well as both encoders receive $\mathbf{Z}_{b-1}$. At this point, encoder 1 makes a list of possible $m_{2(b-1)}$. This list, exponentially smaller than $2^{N R_{2}}$ in size, is resolved by the sequence $\mathbf{B}_{\mathbf{b}}$ that is decoded by encoder 1 at the end of block $b$. Similarly, encoder 2 determines $m_{1(b-1)}$ at the end of block $b$ by decoding $\mathbf{A}_{b}$. At this point, the decoder cannot decode the pair $\left(\mathbf{A}_{b}, \mathbf{B}_{b}\right)$ since it is disadvantaged compared to the encoders. Therefore, at the end of block $b$, it is left with a list of possible message pairs $\left(m_{1(b-1)}, m_{2(b-1)}\right)$. This list is resolved by both encoders together in block $b+1$ using a common sequence $\mathbf{U}_{b+1}$.

Note that $\mathbf{A}_{b}$ and $\mathbf{B}_{b}$ are correlated random sequences since $\left(m_{1(b-1)}, m_{2(b-1)}\right)$ is a correlated message pair [11] given $\mathbf{Z}_{b-1}$. The correlation between $\mathbf{A}_{b}$ and $\mathbf{B}_{b}$ cannot be arbitraryit is generated using the information available at each encoder at the end of block $(b-1)$. At this point, both encoders know $(\mathbf{z}, \mathbf{a}, \mathbf{b}, \mathbf{u}, \mathbf{w})_{b-1}$. In addition, encoder 1 also knows $\mathbf{x}_{b-1}$ and 
generates $\mathbf{A}_{b} \sim Q^{1}\left(. \mid(\mathbf{z}, \mathbf{x}, \mathbf{a}, \mathbf{b}, \mathbf{u}, \mathbf{w})_{b-1}\right)$. Similarly, encoder 2 generates $\mathbf{B}_{b} \sim Q^{2}\left(. \mid(\mathbf{z}, \mathbf{y}, \mathbf{a}, \mathbf{b}, \mathbf{u}, \mathbf{w})_{b-1}\right)$.

In order to obtain a single-letter characterization, we need to mae sure that the $\left(\mathbf{A}_{b}, \mathbf{B}_{b}\right)$ pair has the same joint distribution in each block $b$. This is achieved as follows. $\mathbf{w}_{b}$ is defined as $f\left((\mathbf{z}, \mathbf{a}, \mathbf{b}, \mathbf{u}, \mathbf{w})_{b-1}\right)$, where (3) ensures that $\mathbf{w}_{b} \sim P_{W}$. Then (4) ensures that given $\mathbf{w}_{b}$, the $\left(\mathbf{A}_{b}, \mathbf{B}_{b}\right)$ pair corresponding to each possible $\left(m_{1(b-1)}, m_{2(b-1)}\right)$ is as if picked independently from the typical set $T\left(P_{A B \mid W}\right)$. Conditioned on $\mathbf{w}_{b}$, (4) guarantees that all the sequences corresponding to block $b$ will be independent of all the sequences corresponding to block $b-1, \forall b$. This is crucial for obtaining a single-letter characterization since it avoids sequences over multiple blocks being arbitrarily correlated.

If we set $W=A=B=\phi$, the conditions are trivially satisfied and we obtain the C-L region of Theorem II In the $\mathrm{C}$ - $\mathrm{L}$ coding scheme, the rates $\left(R_{1}, R_{2}\right)$ are low enough so that each encoder can decode the message of the other at the end of the same block. In our strategy, the rates are too high to do this, so the encoders wait one more block to decode their messages (using $\mathbf{A}$ and $\mathbf{B}$ ). In both schemes, the encoders cooperate to resolve the decoders list one block after they decode each other's message.

It is easy to see that our scheme can be extended as follows. Suppose that the rates are high enough so that the correlated information $\mathbf{A}$ and $\mathbf{B}$ cannot be decoded, i.e., the encoders are not able to decode the messages even with a delay of one block. We can then introduce another pair of (correlated) auxiliary random variables that the encoders decode with a delay of two blocks. In other words, we can extend our rate region by introducing an extra pair of auxiliary random variables. Clearly, this can be repeated to obtain potentially larger rate-regions, albeit with more auxiliary random variables.

\section{Proof of Theorem 1}

Due to constraints of space, we only give a brief proof. Fix a joint distribution $P_{U W A B X Y Z}$ as in (2) with corresponding $f, Q^{1}, Q^{2}$ such that (3) and (4) are satisfied.

\section{A. Random Codebook generation}

Choose $2^{N R_{0}}$ sequences $\mathbf{u}[m]$ independently from the typical set $T\left(P_{U}\right), 1 \leq m \leq 2^{N R_{0}}$.

For each $(\mathbf{w}, \mathbf{u}, \mathbf{a}, \mathbf{b}) \in T\left(P_{W U A B}\right)$, generate $2^{N R_{1}}$ sequences $\mathbf{x}[i]$ with $x_{n} \sim P_{X \mid U W A}, 1 \leq i \leq 2^{N R_{1}}$ and $2^{N R_{2}}$ sequences $\mathbf{y}[j]$ with $y_{n} \sim P_{Y \mid U W B}, 1 \leq j \leq 2^{N R_{2}}$.

For each $(\mathbf{z}, \mathbf{x}, \mathbf{a}, \mathbf{b}, \mathbf{u}, \mathbf{w}) \in T\left(P_{Z X A B U W}\right)$, generate one sequence $\overline{\mathbf{a}}$ with $\bar{a}_{n} \sim Q^{1}\left(. \mid z_{n}, x_{n}, a_{n}, b_{n}, u_{n}, w_{n}\right)$. Similarly, for each $(\mathbf{z}, \mathbf{y}, \mathbf{a}, \mathbf{b}, \mathbf{u}, \mathbf{w}) \in T\left(P_{Z Y A B U W}\right)$, generate one sequence $\overline{\mathbf{b}}$ with $\bar{b}_{n} \sim Q^{2}\left(. \mid z_{n}, y_{n}, a_{n}, b_{n}, u_{n}, w_{n}\right)$.

\section{B. Encoding}

For $2 \leq b \leq B$, the encoders generate the inputs $\mathbf{X}_{b}, \mathbf{Y}_{b}$ for block $b$ as follows:

1) As explained later, at the end of block $(b-1)$, both encoders agree on $\mathbf{u}_{b}$ and also know $(\mathbf{z}, \mathbf{a}, \mathbf{b}, \mathbf{u}, \mathbf{w})_{b-1}$. Thus $\mathbf{w}_{b}=f\left((\mathbf{z}, \mathbf{a}, \mathbf{b}, \mathbf{u}, \mathbf{w})_{b-1}\right)$ is also known.
2) Encoder 1 chooses $\mathbf{A}_{b}=\overline{\mathbf{a}}$, where $\overline{\mathbf{a}}$ is the sequence corresponding to $(\mathbf{z}, \mathbf{x}, \mathbf{a}, \mathbf{b}, \mathbf{u}, \mathbf{w})_{b-1}$ (cf. Section $\left.\amalg I I-A\right)$. Similarly, encoder 2 chooses $\mathbf{B}_{b}=\overline{\mathbf{b}}$, with $\overline{\mathbf{b}}$ corresponding to $(\mathbf{z}, \mathbf{y}, \mathbf{a}, \mathbf{b}, \mathbf{u}, \mathbf{w})_{b-1}$.

3) Finally, $(\mathbf{u}, \mathbf{w}, \mathbf{a}, \mathbf{b})_{b}$ determines the codebooks of encoder 1 and 2 , from which $\mathbf{x}_{b}[i]$ and $\mathbf{y}_{b}[j]$ are transmitted if the messages are $i$ and $j$, respectively. No new messages are transmitted in blocks $(B-1)$ and $B$ $\left(\mathbf{x}_{B-1}[1], \mathbf{y}_{B-1}[1]\right)$ and $\left(\mathbf{x}_{B}[1], \mathbf{y}_{B}[1]\right)$ are taken to be the codewords for these two blocks. The reduction in rate due to this is insignificant if $B$ is very large.

For block $1,(\mathbf{u}, \mathbf{w}, \mathbf{a}, \mathbf{b})_{1}$ are chosen a priori at random from $T\left(P_{U W A B}\right)$, and $\mathbf{x}_{1}[i], \mathbf{y}_{1}[j]$ are transmitted from the respective codebooks, if $(i, j)$ are the messages in block 1 .

\section{Decoding}

Upon receiving $\mathbf{z}_{b}$, the decoder decodes the message pair corresponding to block $(b-2)$, while the encoders decode the messages (of one another) corresponding to block $(b-1)$. This is explained below.

At the Decoder $(3 \leq b \leq B)$ : At the end of block $(b-1)$,

- the decoder has correctly decoded $\mathbf{u}_{b-1}$ from $\mathbf{z}_{b-1}$,

- it knows the tuple $(\mathbf{z}, \mathbf{a}, \mathbf{b}, \mathbf{u}, \mathbf{w})_{b-2}$ (thus also $\mathbf{w}_{b-1}$ ).

At this point, the decoder has the following list of all possible message pairs for block $(b-2)$ :

$$
\begin{array}{r}
\mathcal{L}_{b-2} \triangleq\left\{(i, j):(\mathbf{z}, \mathbf{x}[i], \mathbf{y}[j], \mathbf{a}, \mathbf{b}, \mathbf{u}, \mathbf{w})_{b-2}\right. \text { jointly typ. } \\
\left.\operatorname{AND}\left(\mathbf{z}_{b-1}, \mathbf{a}_{i}, \mathbf{b}_{j}, \mathbf{u}_{b-1}, \mathbf{w}_{b-1}\right) \text { jointly typ. }\right\}
\end{array}
$$

where $\mathbf{a}_{i}$ denotes the $\overline{\mathbf{a}}$-sequence corresponding to $(\mathbf{z}, \mathbf{x}[i], \mathbf{a}, \mathbf{b}, \mathbf{u}, \mathbf{w})_{b-2}$, and $\mathbf{b}_{j}$ denotes the $\overline{\mathbf{b}}$-sequence corresponding to $(\mathbf{z}, \mathbf{y}[j], \mathbf{a}, \mathbf{b}, \mathbf{u}, \mathbf{w})_{b-2}$ (cf. Section $\left.\amalg I-A\right)$.

Upon receiving $\mathbf{z}_{b}$, the decoder determines the unique $m^{*} \in$ $\left\{1, \ldots, 2^{N R_{0}}\right\}$ such that $\left(\mathbf{z}_{b}, \mathbf{u}\left[m^{*}\right]\right)$ is jointly typical. This is done correctly with high probability if

$$
R_{0}<I(U ; Z)-\epsilon
$$

If the list-size $\left|\mathcal{L}_{b-2}\right|$ is smaller than $2^{N R_{0}}$, the decoder can resolve it using $m^{*}$. Assuming $\left(i^{*}, j^{*}\right)$ is the message pair transmitted, we have $\mathcal{L}_{b-2}=\left\{\left(i^{*}, j^{*}\right)\right\} \cup \mathcal{L}_{1} \cup \mathcal{L}_{2} \cup \mathcal{L}_{3}$, where $\mathcal{L}_{1} \triangleq\left\{\left(i^{*}, j\right), j \neq j^{*}:\left(\mathbf{z}, \mathbf{x}\left[i^{*}\right], \mathbf{y}[j], \mathbf{a}, \mathbf{b}, \mathbf{u}, \mathbf{w}\right)_{b-2}\right.$ jointly typ. $\operatorname{AND}\left(\mathbf{z}_{b+1}, \mathbf{a}_{i^{*}}, \mathbf{b}_{j}, \mathbf{u}_{b+1}, \mathbf{w}_{b-1}\right)$ are jointly typ.\},

$\mathcal{L}_{2} \triangleq\left\{\left(i, j^{*}\right), i \neq i^{*}:\left(\mathbf{z}, \mathbf{x}[i], \mathbf{y}\left[j^{*}\right], \mathbf{a}, \mathbf{b}, \mathbf{u}, \mathbf{w}\right)_{b-2}\right.$ jointly typ. $\operatorname{AND}\left(\mathbf{z}_{b+1}, \mathbf{a}_{i}, \mathbf{b}_{j^{*}}, \mathbf{u}_{b+1}, \mathbf{w}_{b-1}\right)$ are jointly typ.\},

$\mathcal{L}_{3} \triangleq\left\{(i, j), i \neq i^{*}, j \neq j^{*}:(\mathbf{z}, \mathbf{x}[i], \mathbf{y}[j], \mathbf{a}, \mathbf{b}, \mathbf{u}, \mathbf{w})_{b-2}\right.$ jointly typ. AND $\left(\mathbf{z}_{b+1}, \mathbf{a}_{i}, \mathbf{b}_{j}, \mathbf{u}_{b+1}, \mathbf{w}_{b-1}\right)$ are jointly typ.\},

Consider $\mathcal{L}_{1}$. The probability of $\left(\mathbf{z}, \mathbf{x}\left[i^{*}\right], \mathbf{y}[j], \mathbf{a}, \mathbf{b}, \mathbf{u}, \mathbf{w}\right)_{b-2}$ being jointly typical can be upper-bounded by $2^{-N(I(Y ; Z \mid X A B W U)-\epsilon)}$. Conditioned on $\mathbf{w}_{b-1}$, note that all the sequences in block $b-1$ are independent of all the sequences in block $b-2$. Since $\mathbf{w}_{b-1}$ is known at both encoders at the beginning of block $b-1$, the probability 
of $\left(\mathbf{z}_{b+1}, \mathbf{a}_{i^{*}}, \mathbf{b}_{j}, \mathbf{u}_{b+1}, \mathbf{w}_{b-1}\right)$ being jointly typical, given $\left(\mathbf{z}, \mathbf{x}\left[i^{*}\right], \mathbf{y}[j], \mathbf{a}, \mathbf{b}, \mathbf{u}, \mathbf{w}\right)_{b-2}$ is jointly typical, can be upper-bounded by $2^{-N(I(B ; Z \mid X A W U)-\epsilon)}$. We therefore have

$$
E\left|\mathcal{L}_{1}\right|<2^{N R_{2}} 2^{-N(I(Y ; Z \mid X A B U W)-\epsilon)} 2^{-N(I(B ; Z \mid A U W)-\epsilon)}
$$

Using similar upper bounds for $E\left|\mathcal{L}_{2}\right|$ and $E\left|\mathcal{L}_{3}\right|$, we obtain

$$
\begin{aligned}
E\left|\mathcal{L}_{b-2}\right|< & 1+2^{N\left(R_{2}-I(Y ; Z \mid X A B U W)-I(B ; Z \mid A U W)+2 \epsilon\right)} \\
& +2^{N\left(R_{1}-I(X ; Z \mid Y A B U W)-I(A ; Z \mid B U W)+2 \epsilon\right)} \\
& +2^{N\left(R_{1}+R_{2}-I(X Y ; Z \mid U W)+2 \epsilon\right)}
\end{aligned}
$$

Using (7) in the Markov inequality, one can show that with high probability, $\left|\mathcal{L}_{b-2}\right|<2^{N(A+4 \epsilon)}$, where

$$
\begin{aligned}
A \triangleq & \max \left\{R_{1}+R_{2}-I(X Y ; Z \mid U W),\right. \\
& R_{1}-I(X ; Z \mid Y A B U W)-I(A ; Z \mid B U W), \\
& \left.R_{2}-I(Y ; Z \mid X A B U W)-I(B ; Z \mid A U W)\right\} .
\end{aligned}
$$

Thus $\mathbf{U}_{b}=\mathbf{u}\left[m^{*}\right]$ can resolve the list $\mathcal{L}_{b-2}$ if $2^{N(A+4 \epsilon)}<$ $2^{N R_{0}}$. Combining this with (6), we conclude that $m^{*}$ can uniquely index the correct message pair $\left(i^{*}, j^{*}\right)$ in $\mathcal{L}_{b-2}$ if

$$
\begin{aligned}
R_{1}+R_{2}-I(X Y ; Z \mid U W) & <I(U ; Z)-5 \epsilon, \\
R_{1}-I(X ; Z \mid Y A B U W)-I(A ; Z \mid B U W) & <I(U ; Z)-5 \epsilon, \\
R_{2}-I(Y ; Z \mid X A B U W)-I(B ; Z \mid A U W) & <I(U ; Z)-5 \epsilon .
\end{aligned}
$$

The decoder now also knows the tuple $(\mathbf{z}, \mathbf{a}, \mathbf{b}, \mathbf{u}, \mathbf{w})_{b-1}$ since $\mathbf{a}_{b-1}=\mathbf{a}_{i^{*}}$ and $\mathbf{b}_{b-1}=\mathbf{b}_{j^{*}}$. This is the information required for decoding at the end of the next block $b+1$.

At the Encoders $(2 \leq b \leq B)$ : At the end of block $(b-1)$, the encoders agree on a common $\mathbf{u}_{b}$ and also know $(\mathbf{z}, \mathbf{a}, \mathbf{b}, \mathbf{u}, \mathbf{w})_{b-1}$ and $\mathbf{w}_{b}=f\left((\mathbf{z}, \mathbf{a}, \mathbf{b}, \mathbf{u}, \mathbf{w})_{b-1}\right)$. In the next block, $\mathbf{z}_{b}$ is received. Let $\mathbf{b}_{j}$ denote the $\overline{\mathbf{b}}$-sequence corresponding to $(\mathbf{z}, \mathbf{y}[j], \mathbf{a}, \mathbf{b}, \mathbf{u}, \mathbf{w})_{b-1}, 1 \leq j \leq 2^{N R_{2}}$. If there is a unique $j^{*}$ such that $\left(\mathbf{z}_{b}, \mathbf{x}_{b}, \mathbf{a}_{b}, \mathbf{b}_{j^{*}}, \mathbf{u}_{b}, \mathbf{w}_{b}\right)$ is jointly typical, encoder 1 decodes $j^{*}$ to be the message sent by encoder 2 in block $(b-1)$. An error is made only if there exists $j \neq j^{*}$ such that $(\mathbf{z}, \mathbf{y}[j], \mathbf{a}, \mathbf{b}, \mathbf{u}, \mathbf{w})_{b-1}$ and $\left(\mathbf{z}_{b}, \mathbf{x}_{b}, \mathbf{a}_{b}, \mathbf{b}_{j}, \mathbf{u}_{b}, \mathbf{w}_{b}\right)$ are each jointly typical. The probability of this event can be upper bounded by

$$
\sum_{j=1}^{2^{N R_{2}}} 2^{-N(I(Y ; Z \mid X A B W U)-\epsilon)} 2^{-N(I(B ; Z \mid X A W U)-\epsilon)} .
$$

A similar upper bound can be obtained for the probability of encoder 2 wrongly decoding $i^{*}$, the message sent by encoder 1 in block $(b-1)$. Hence the probability of each encoder decoding the other's message incorrectly can be made arbitrarily small if

$$
\begin{aligned}
R_{2}+2 \epsilon & <I(Y ; Z \mid X A B W U)+I(B ; Z \mid X A W U) \\
& =I(Y B ; Z \mid X A W U)=I(Y ; Z \mid X A W U), \\
R_{1}+2 \epsilon & <I(X ; Z \mid Y A B W U)+I(A ; Z \mid Y B W U) \\
& =I(X A ; Z \mid X B W U)=I(X ; Z \mid Y B W U) .
\end{aligned}
$$

At this point, the decoder has a list $\mathcal{L}_{b-1}$ of possible message pairs for block $(b-1)$ (cf. (5)). The encoders both set $\mathbf{U}_{b+1}=\mathbf{u}\left[m^{*}\right]$, where $m^{*} \in\left\{1, \ldots, 2^{N R_{0}}\right\}$ uniquely determines $\left(i^{*}, j^{*}\right) \in \mathcal{L}_{b-1}$. Note that if no error is made by the encoders in decoding $i^{*}, j^{*}$ at the end of block $b$, both encoders know $\left(\mathbf{a}_{b}, \mathbf{b}_{b}\right)$ to be equal to $\left(\mathbf{a}_{i^{*}}, \mathbf{b}_{j^{*}}\right)$, as required for the next block.

Probability of Error: Using (4), one can show that $(\mathbf{u}, \mathbf{w}, \mathbf{a}, \mathbf{b}, \mathbf{x}, \mathbf{y}, \mathbf{z})_{b}$ is jointly typical according to (2) with high probability $\forall b$. Further, we have shown that the probability of error at the decoder and each encoder can be made arbitrarily small if (8) and (9) are satisfied.

\section{EXAMPLE}

Consider the AWGN MAC with power constraint $P$ on each of the inputs. This channel, with $\mathcal{X}=\mathcal{Y}=\mathcal{Z}=\mathbb{R}$, is defined by

$$
Z=X+Y+N
$$

where $N$ is a Gaussian noise random variable with mean 0 and variance $\sigma^{2}$ that is independent of $X$ and $Y$. The inputs $\mathbf{x}$ and y for each block satisfy $\frac{1}{N} \sum_{n=1}^{N} x_{n}^{2} \leq P, \frac{1}{N} \sum_{n=1}^{N} y_{n}^{2} \leq P$. For this channel, the equal-rate point on the boundary of the C-L region [1] is $\left(R_{C L}, R_{C L}\right)$ where

$$
R_{C L}=\frac{1}{2} \log \left(2 \sqrt{1+\frac{P}{\sigma^{2}}}-1\right)
$$

The achievable rate region of Theorem 1 for the discrete memoryless case can be extended to the AWGN MAC using a similar proof, recognizing that in the Gaussian case superposition is equivalent to addition.

For the joint distribution $P_{U W A B X Y Z}$ in (2), define $U \sim$ $\mathcal{N}(0,1)$ and $(W, A, B)$ jointly Gaussian with mean zero and covariance matrix

$$
K_{W A B}=\left[\begin{array}{ccc}
1 & \gamma & -\gamma \\
\gamma & 1 & \lambda \\
-\gamma & \lambda & 1
\end{array}\right]
$$

The input distributions $P_{X \mid U W A}$ and $P_{Y \mid U W B}$ are defined by

$$
\begin{aligned}
& X=\sqrt{\alpha P} I_{X}+\sqrt{\beta P} A+\sqrt{\overline{\alpha+\beta} P} U, \\
& Y=\sqrt{\alpha P} I_{Y}+\sqrt{\beta P} B+\sqrt{\overline{\alpha+\beta} P} U
\end{aligned}
$$

where $I_{X}, I_{Y}$ are independent $\mathcal{N}(0,1)$ random variables, $\alpha, \beta>0$ and $\alpha+\beta \leq 1 . I_{X}$ and $I_{Y}$ represent the fresh information and $U$ is the resolution information for the decoder that the encoders cooperate to send. $A$ is the information that encoder 2 decodes using feedback, and $B$ the information that encoder 1 decodes. We need to verify that the two conditions of Theorem 1 are satisfied by this joint distribution and then evaluate the rates.

\section{A. Verifying the conditions}

At the end of block $b$, both encoders know $(\mathbf{Z}, \mathbf{A}, \mathbf{B}, \mathbf{U}, \mathbf{W})_{b}$. This tuple is represented as $\mathbf{W}_{b+1}$ 
TABLE I

COMPARISON OF EQUAL-RATE BOUNDARY POINTS

\begin{tabular}{|c|c|c|c|c|c|}
\multicolumn{6}{|c|}{$P / \sigma^{2}$} \\
\hline & 0.5 & 1 & 5 & 10 & 100 \\
\hline$R_{C L}$ & 0.2678 & 0.4353 & 0.9815 & 1.2470 & 2.1277 \\
$R^{*}$ & 0.2754 & 0.4499 & 1.0067 & 1.2709 & 2.1400 \\
$R_{\text {FBcap }}$ & 0.2834 & 0.4642 & 1.0241 & 1.2847 & 2.1439 \\
\hline
\end{tabular}

in the next block $b+1$ using a function $f\left((\mathbf{Z}, \mathbf{A}, \mathbf{B}, \mathbf{U}, \mathbf{W})_{b}\right)$. Define the function as

$$
\begin{aligned}
\bar{W} & =f(Z, A, B, U) \\
& \triangleq \frac{Z-\sqrt{\beta P} A-\sqrt{\beta P} B-2 \sqrt{\overline{\alpha+\beta} P} U}{\sqrt{2 \alpha P+\sigma^{2}}} .
\end{aligned}
$$

From (13) and (10), we see that $f$ removes the effect of $(\mathbf{U}, \mathbf{A}, \mathbf{B})_{b}$ from $\mathbf{Z}_{b}$, resulting in a $\mathbf{W}_{b+1}$ that is the sum of the fresh information in block $b$ and the channel noise.

Sequences $\mathbf{A}_{b+1}$ and $\mathbf{B}_{b+1}$ are generated using $\mathbf{X}_{b}$ and $\mathbf{Y}_{b}$, respectively, along with the common tuple $(\mathbf{Z}, \mathbf{A}, \mathbf{B}, \mathbf{U}, \mathbf{W})_{b}$. Define the following functions to generate $(\mathbf{A}, \mathbf{B})_{b+1}$ :

$q_{1}: \bar{A}=k_{1} \frac{X-\sqrt{\overline{\alpha+\beta} P} U-\sqrt{\beta P} A}{\sqrt{\alpha P}}+k_{2} f(U, A, B, Z)$,
$q_{2}: \bar{B}=-k_{1} \frac{Y-\sqrt{\overline{\alpha+\beta} P} U-\sqrt{\beta P} B}{\sqrt{\alpha P}}-k_{2} f(U, A, B, Z)$

where $k_{1}, k_{2} \in \mathbb{R}$. With respect to (4), this is equivalent to

$$
\begin{aligned}
& Q^{1}(\bar{a} \mid z, x, a, b, u, w)=\mathbf{1}\left(q_{1}(z, x, a, b, u, w)=\bar{a}\right), \\
& Q^{2}(\bar{b} \mid z, y, a, b, u, w)=\mathbf{1}\left(q_{2}(z, y, a, b, u, w)=\bar{b}\right) .
\end{aligned}
$$

(15) ensures that $(\mathbf{A}, \mathbf{B})_{b+1}$ depend on $(\mathbf{Z}, \mathbf{A}, \mathbf{B}, \mathbf{U})_{b}$ only through ' $\mathbf{Z}_{b}$ with the effect of $(\mathbf{A}, \mathbf{B}, \mathbf{U})_{b}$ removed'. In other words, conditioned on $\mathbf{W}_{b+1}=f\left((\mathbf{Z}, \mathbf{A}, \mathbf{B}, \mathbf{U})_{b}\right),(\mathbf{A}, \mathbf{B})_{b+1}$ is independent of all the sequences in block $b$ as required by (4). Indeed, it can be formally verified that (4) holds if the following equalities are satisfied.

$E\left[\bar{A}^{2}\right]=E\left[\bar{B}^{2}\right]=1, E[\bar{A} \bar{B}]=\lambda, E[\bar{A} \bar{W}]=-E[\bar{B} \bar{W}]=\gamma$

Evaluating the conditions in (16) using (14) and (15), we have

$$
\begin{gathered}
1=E\left[\bar{A}^{2}\right]=k_{1}^{2}+k_{2}^{2}+2 k_{1} k_{2} \sqrt{\frac{\alpha P}{2 \alpha P+\sigma^{2}}}, \\
\lambda=E[\bar{A} \bar{B}]=-k_{2}^{2}-2 k_{1} k_{2} \sqrt{\frac{\alpha P}{2 \alpha P+\sigma^{2}}}, \\
\gamma=E[\bar{A} \bar{W}]=k_{2}+k_{1} \sqrt{\frac{\alpha P}{2 \alpha P+\sigma^{2}}} .
\end{gathered}
$$

Adding (17) and (18), we get $k_{1}^{2}=1+\lambda$. Substituting each value of $k_{1}$ in (17) yields a quadratic equation that can be solved to obtain $k_{2}$. Finally $\gamma$ is determined from (19) using $k_{1}$ and $k_{2}$. To summarize, once we choose $(\alpha, \beta, \lambda)$, the value of $\gamma$ is determined by (19) in order for the joint distribution to be valid for Theorem 1

\section{B. Evaluating the rates}

For a valid $(\alpha, \beta, \lambda, \gamma)$, the achievable rates can be evaluated from Theorem 1 to be

$$
\begin{aligned}
R_{1}, R_{2} & <\min \{G, H\}, \\
R_{1}+R_{2} & <\frac{1}{2}\left(1+\frac{2 P}{\sigma^{2}}+\frac{2 P}{\sigma^{2}}(\overline{\alpha+\beta}+\lambda \beta)\right),
\end{aligned}
$$

where

$$
\begin{aligned}
G=\frac{1}{2} \log (1 & +(\alpha+\beta) \frac{P}{\sigma^{2}} \\
- & \left.\beta \frac{P}{\sigma^{2}} \cdot \frac{\lambda^{2}+\gamma^{2}\left(1-\lambda^{2}+2 \lambda\right)-\gamma^{4}(1+2 \lambda)}{\left(1-\gamma^{2}\right)^{2}}\right), \\
H=\frac{1}{2} \log (1+ & \left.\frac{4 \overline{\alpha+\beta} P / \sigma^{2}}{2(\alpha+\beta+\beta \lambda) P / \sigma^{2}+1}\right)+\frac{1}{2} \log \left(1+\alpha \frac{P}{\sigma^{2}}\right) \\
& +\frac{1}{2} \log \left(1+\frac{\beta(1+\lambda) P / \sigma^{2}}{1+2 \alpha P / \sigma^{2}}\left(2-\frac{1+\lambda}{1-\gamma^{2}}\right)\right) .
\end{aligned}
$$

For different values of $\frac{P}{\sigma^{2}}$, we (numerically) compute the equal-rate point $\left(R^{*}, R^{*}\right)$ on the boundary of (20). For various values of the signal-to-noise ratio $P / \sigma^{2}$, Table $\llbracket$ compares $R^{*}$ with $R_{C L}$, the equal-rate point of the C-L region given by (11), and with the equal rate-point $R_{\mathrm{FBcap}}$ on the boundary of the feedback capacity region [6]. We observe that our equalrate points represent a significant improvement over the C-L region, and are close to the feedback capacity for large SNR.

It is part of ongoing work to compare our rate region with that of [9]. Though direct comparison of the two regions appears difficult in general, it may be possible for specific channels. Moreover, as explained at the end of Section $\Pi$ our strategy can be extended to yield larger rate-regions by adding more auxiliary random variables.

\section{REFERENCES}

[1] T. M. Cover and C. S. K. Leung, "An achievable rate region for the multiple-access channel with feedback," IEEE Trans. Inf. Theory, vol. 27, no. 3, pp. 292-298, 1981.

[2] R. Ahlswede, "Multi-way communication channels," in Proc. Second Int. Symp. Inform. Transmission, Tsahkadsor, Armenia, USSR, Hungarian Press, 1971

[3] H. D. Liao, Multiple-Access Channels. PhD thesis, Univ. Hawaii, 1972.

[4] N. T. Gaarder and J. K. Wolf, "The capacity region of a multiple-access discrete memoryless channel can increase with feedback," IEEE Trans. on Inf. Theory, vol. 21, no. 1, pp. 100-102, 1975.

[5] F. M. J. Willems, "The feedback capacity region of a class of discrete memoryless multiple access channels," IEEE Trans. Inf. Theory, vol. 28, no. 1, pp. 93-95, 1982.

[6] L. H. Ozarow, "The capacity of the white gaussian multiple access channel with feedback," IEEE Trans. Inf. Theory, vol. 30, no. 4, pp. 623$628,1984$.

[7] G. Kramer, Directed Information for channels with Feedback. PhD thesis, Swiss Federal Institute of Technology, Zurich, 1998.

[8] G. Kramer, "Capacity Results for the Discrete Memoryless Network," IEEE Transactions on Information Theory, vol. 49, pp. 4-20, January 2003.

[9] S. Bross and A. Lapidoth, "An improved achievable region for the discrete memoryless two-user multiple-access channel with noiseless feedback," IEEE Trans Inf Theory, vol. IT-51, pp. 811-833, March 2005.

[10] T. M. Cover, A. El Gamal, and M. Salehi, "Multiple access channels with arbitrarily correlated sources," IEEE Trans. Inf. Theory, vol. 26, no. 6, pp. 648-657, 1980.

[11] S. S. Pradhan, S. Choi, and K. Ramchandran, "A graph-based framework for transmission of correlated sources over multiple-access channels," IEEE Trans Inf Theory, vol. 53, no. 12, pp. 4583-4604, 2007. 